\title{
Relating Vegetation Aerodynamic Roughness Length to Interferometric SAR Measurements
}

\author{
Sassan Saatchi and Emesto Rodriguez \\ Jet Propulsion Laboratory, California Institute of Technology, Pasadena, California 91109
}

\section{Abstract}

In this paper, we investigate the feasibility of estimating aerodynamic roughness parameter from interferometric SAR (INSAR) measurements. The relation between the interferometric correlation and the rms height of the surface is presented analytically. Model simulations performed over realistic canopy parameters obtained from field measurements in boreal forest environment demonstrate the capability of the INSAR measurements for estimating and mapping surface roughness lengths over forests and/or other vegetation types. The procedure for estimating this parameter over boreal forests using the INSAR data is discussed and the possibility of extending the methodology over tropical forests is examined.

\section{Introduction}

Realistic models of land surfaceatmosphere interactions were introduced into general circulation models of the atmosphere (GCMs) in the mid-1980 (Dickinson, 1984, (Sellers et al., 1986). These land surface parameterization were based on simple formulations of the processes of radiative transfer (albedo), turbulent transfer (roughness length) and evapotranspiration (surface resistance) associated with vegetated surfaces to describe the exchanges of energy, momentum, and sensible and latent heat, respectively, between the vegetation-soil system and the lowest atmosphere layer of a GCM. In general these models suffer from several shortcomings, particularly, the paucity of global data on some input parameters. Currently, the GCM models function at a coarse resolution using $1^{\circ}$ by $1^{\circ}$ grid data, but the submodels (mesoscale) operate at smaller resolution and therefore require finer scale data sets. This lack of data of surface parameters such as vegetation has limited our understanding of climate change/variability and its relation with the status and dynamics of the biotic changes of land surface. With the initiative of the NASA's Mission to Planet Earth (MTPE) program, several studies have been carried out within the earth science disciplines such as ISLSCP and large scale field experiments (FIFE, HAPEX, BOREAS, LBA). All these studies recognize that satellite data offers the most economic and consistent means to provide some of the data required by modelers at various scales (Sellers et al., 1994). So far the main source of data for deriving the required parameters for land-surface atmospheric and climate studies has been the AVHRR instrument. Sellers et al., (1994) outline the estimation of these parameters from the AVHRR data. Three essential parameters: albedo, surface roughness, and surface conductance, were derived from the AVHRR $1^{\circ}$ by $1^{\circ}$ normalized difference vegetation index (NDVI). In addition, the same data set was used to derive land cover types and other vegetation characteristics such as FPAR (Fraction of Photosyntheticaly Active Radiation), LAI (Leaf Area Index) which were used directly in GCM or SiB2 (Simple Biosphere Models).

\section{Aerodynamic Roughness}

The aerodynamic roughness length $Z_{0}$ is defined as the height where the wind speed becomes zero. In modeling the wind profile and drag over vegetation canopies, the height where the wind speed becomes zero inside the canopy layer is referred as the aerodynamic roughness length of the vegetation. The roughness length appears explicitly in the logarithmic wind-profile equation (Monteith and Unsworth, 1990). This parameter is entirely determined by the structure of vegetation, i.e. the disposition of the biomass in 3-D space. There are many empirical relationships that relate $Z_{0}$ to vegetation height. 
But, the relationship should also include the density or the spacing of vegetation elements. The relationship, therefore, appears to be nonlinear (logarithmic) such that for a vegetated surface at a given height, the $Z_{0}$ increases as the density increases, till it reaches a maximum and then decreases gradually (similar arguments are applied for non-vegetated surfaces). This phenomena is not difficult to interpret. When the density of sparsely-placed surface elements (e.g. vegetation components) increases, the drag increases and therefore, also $Z_{0}$; but when the placement of elements becomes extremely dense, the flow of wind may actually skim over the top of the canopy without entering the space below. Given the logarithmic relationship, $Z_{0}$ can be obtained by measuring the wind speed at two or more heights. Once this roughness length is determined for a certain surface, it does not change with wind speed, stability or stress. However, it can change if the structure and density of surface roughness elements change (e.g. because of land cover change, deforestation, soil erosion, etc.).

Although aerodynamic roughness length is not equal to the absolute height of individual roughness elements on the ground, there is a relationship between them (Brutsaert, 1991). This relationship appears to be more direct with the physical roughness of the surface (height variations) than the absolute height. For most surfaces, the physical roughness follows the same behavior as the aerodynamic roughness with respect to the density of surface elements (Blumberg and Greeley, 1993).

Typically, $Z_{0}$ is derived from measurements of the wind velocity profile through the lower atmosphere boundary-layer. Such measurements require elaborate field equipment, including arrays of anemometers on masts, temperature probes, and recording equipment. Moreover, $Z_{0}$ values for natural surfaces obtained in this way are limited to immediate sites where such measurements are made. Consequently, very little data on aerodynamic roughness have been obtained, despite the relevance for climate studies and analysis of land-surface atmospheric interactions.

\section{Methodology}

To remove the uncertainties of the aerodynamic roughness parameter in boundary- layer models, we propose a methodology to use the interferometric SAR data to accurately measure the physical roughness or height variation of the surface. By using a simple one-to-one relationship between physical roughness and the aerodynamic roughness, this parameter can be mapped accurately over various land cover types.

The interferometric correlation (i.e., the correlation coefficient between the two interferometric channels-a byproduct of the standard interferometric processing) contains useful information regarding vegetation canopies. Departures from perfect correlation are due to differences in the radar signal between the two interferometric channels. These differences can be due to different thermal noise in the two channels; or more importantly, the slightly different viewing geometry for each channel causes additional deceleration in the two signals. Part of this additional decorelation is due to the angular spread of the radar resolution cell; i.e., the well known van Cattiest Zernike theorem [Goodman, 1985]. It has been shown theoretically [Rodriguez and Martin, 1992], [Treuhaft et al., 1997], [Rodriguez et al., 1995] that an additional amount of decorelation can be related to the amount of volumetric scattering within the radar resolution cell. This volumetric scattering can be due to either penetration into the canopy, or to the fact that the canopy itself presents a rough interface, giving rise to dispersion in the phase [Rodriguez and Martin, 1992]. It is this effect that we propose to use to estimate the aerodynamic roughness.

To demonstrate this, we used a physically based model for forest canopies for interferometric SAR measurements and developed a relationship between the dispersion relationship introduced by Rodriguez and Martin and vegetation parameters (Saatchi and McDonald, 1997). The model was then used to simulate several forest types such as conifers and deciduous stands in boreal forests and examined the sensitivity of SAR correlation as a function of canopy parameters. Based on these results a simple algorithm has been developed to estimate the vegetation height variation and roughness parameter.

\section{Discussion}

As a validation of this height variance estimation, we have used the data from two 
cxperiments: (1) JPL TOPSAR C-band $(5.6 \mathrm{~cm}$ wavclength) interferometer and the Goddard laser profilometer over coniferous forest in the Guifford-Pinchot National Forest, WA, and (2) JPL TOPSAR data and ground data over "the BOREAS study area near Prince Albert, Canada. The scenes studied contained a variety of canopy types, ranging from mature heterogeneous coniferous forests, to homogeneous stands at various stages of regrowth.

The results showed a strong correlation between laser and TOSAR measurements of height variance, indicating that the interferometer and laser have similar penetration characteristics into the canopy; i.e., geometric blockage due to either tree height variations or the canopy gap structure are the dominant mechanisms governing the scattering. The estimation algorithm for TOPSAR data also showed a correlation with the vegetation type. This implied that the estimation accuracy of the vegetation height variance can be improved by modifying the algorithm for various forest types readily extracted from land cover maps.

Another important issue in improving the estimation accuracy is the number of pixels (resolution) used in estimating the height variance. This difference in number of pixels or resolution gives rise to different amounts of decorelation due to the van-Cittert Zemike effect. However, this effect is deterministic once the scattering geometry is known, and can be removed using the interferometric data itself [Rodriguez et al, 1999]. Detailed simulations using the TOPSAR data at degraded resolutions have shown that the main effect in this degradation in resolution is to increase the random noise in the height variance measurement. In order to obtain sensitivities of the order of few centimeter to the height variance, it is necessary to degrade the resolution of the estimated roughness.

\section{Acknowledgment}

This work has been performed at the Jet Propulsion Laboratory, California Institute of technology under a grand from National Aeronautics and Space Administration.
Blumberg, D.G. and R. Greeley, 1993. Field studies of aerodynamic roughness, J. Arid Environ., vol. 25, 39-48.

Dickinson, R.E., $1984 . \quad$ Modeling evapotranspiration for three dimensional global climate models, Climate Processes and Climate Sensitivity, edited by I.E. Hansen and T. Takahashi, Geophysical Monographs, American Geophysical Union, 29, 58-72.

Goodman, J. W., 1985. Statistical Optics, WileyInterscience, New York.

Rodriguez, E., and Martin, J.M., 1992. "Theory and design of interferometric SAR", IEE Proceedings- $F, 139,2$, 147-159.

Rodriguez, E., Michel, T.R., Harding, D., 1998. "Interferometric measurement of canopy height characteristics for coniferous forests," Radio Science, Submitted.

Saatchi, S. and K. McDonnald, 1997, "Coherent effects in microwave backscattering models for forest canopies," IEEE Trans. Geasci. Remote Sensing, Vol. 35, No. 3, 585-598.

Sellers, P. J., Y. Mintz, Y. C. Sud, and A. Dalcher, A simple biosphere model (SiB) for use within general circulation models, J. Atmos. Sci., 43, 505-531, 1986.

Sellers, P.J. C.J. Tucker, G.J. Collatz, S.O. Los, C.O. Justice, D.A. Dazlich and H.E. Dregne, 1994. A global $1^{\circ}$ by $1^{\circ} \mathrm{NDVI}$ data set for climate studies. Part 2: The generation of global fields of terrestrial biophysical parameters from the NDVI, Int. J. Remote Sens., vol. 15, 3519. 3547.

Treuhaft, R.N. and Madsen, S.N, and Moghaddam, M. and vanZyl, J.J., 1997, "Interferometric remote sensing of vegetation and surface topography", Radio Science, 39(6), 14491485.

\section{References}

